\title{
Patients' values and other decisional factors regarding treatment of hypercalcemia of malignancy: A Systematic Review Protocol
}

\author{
Aya Bassatne \\ American University of Beirut Medical Center https://orcid.org/0000-0002-7841-3986 \\ Maya Rahme \\ American University of Beirut Medical Center \\ Thomas Piggott \\ McMaster University \\ Mohammad H Murad \\ Mayo Clinic Minnesota \\ Layal Hneiny \\ American University of Beirut \\ Ghada El-Hajj Fuleihan ( $\square$ gf01@aub.edu.lb )
}

\section{Protocol}

Keywords: Drug, Adults, Hypercalcemia of malignancy, Decision, Cost, Effectiveness, Values, Preferences, Systematic review

Posted Date: January 22nd, 2021

DOI: https://doi.org/10.21203/rs.3.rs-64830/v2

License: (우 (i) This work is licensed under a Creative Commons Attribution 4.0 International License. Read Full License 


\section{Abstract}

Background: Hypercalcemia of malignancy is the second most common cause of hypercalcemia and is associated with significant morbidity and mortality. Several treatment options are available including pharmacological therapy with bisphosphonates, denosumab, glucocorticoids, and calcimimetics, as well as conventional therapy with hydration and possibly calcitonin. While guidelines have previously considered treatment effects, no guideline has yet considered a range of contextual factors impacting recommendations for the management.

Objective: The aim of this study is to summarize the available evidence on important decisional factors for the development of guidelines for the treatment of hypercalcemia of malignancy. These include patient's values and preferences, cost, acceptability, feasibility, and equity.

Methods/Design: This is a systematic review of observational studies, case series, trials, reviews and qualitative studies involving treatment of adult patients with hypercalcemia of malignancy. We developed and executed two independent search strategies using five databases: Pubmed, Medline (OVID), Embase.com, CINAHL (EBSCO) and Cochrane, and reviewed their combined output. Two reviewers screened titles and abstracts and full texts and will implement data abstraction from relevant studies independently and in duplicate. The outcomes of interest are the decisional factors that influence drug selection, with possible subgroup analyses by drug class or etiology of hypercalcemia of malignancy. We will present the data collected in a narrative and thematic approach.

Discussion: This systematic review will identify important treatment decisional factors, and will assist guidelines panels, physicians and patients to decide on therapeutic options based on the current evidence.

\section{Background}

Hypercalcemia affects 1 to $2 \%$ of the general population. Hypercalcemia of malignancy (HCM) is considered the second most common cause of hypercalcemia, after hyperparathyroidism in adults (1). In fact, one third of cancer patients will eventually experience hypercalcemia with the most common causes being breast cancer, lung cancer and multiple myeloma $(2,3)$. HCM arises due to four main mechanisms: humoral secretion of parathyroid hormone related peptide (PTHrP) accounts for over $80 \%$ of cases and occurs most commonly in breast cancer and squamous cell carcinoma of the lung, head and neck, and the kidney, local osteolytic release of calcium, known as local osteolytic hypercalcemia (LOH), such as seen with multiple myeloma and some breast cancers, high levels of calcitriol (1,25-dihydroxyvitamin D) such as noted in leukemias, HTLV1, and some lymphomas, or secretion of the native parathyroid hormone (PTH) from a carcinoma, or ectopic PTH secretion by some cancers including neuroendocrine tumors (Table 1) $(4,5)$. These include tumors in the head and neck, thorax, gastrointestinal system, or genito-urinary system (6). Hypercalcemia can be classified into mild, moderate or severe. Although mild hypercalcemia can be asymptomatic, moderate and severe hypercalcemia can be associated with a wide range of symptoms from polyuria, polydipsia, dehydration, nephrolithiasis, and muscle weakness all the way to renal failure, lethargy, coma, and cardiac arrest (4). Although not very common, HCM is associated with a longer hospital stay and 
greater mortality risk when compared to cancer patients without $\mathrm{HCM}(7)$. In fact, $50 \%$ of patients with $\mathrm{HCM}$ may die within a month $(7,8)$. Therefore, treatment is of utmost importance.

Treatment of hypercalcemia of malignancy constitutes of hydration, calciuresis and inhibition of bone resorption $(4,8)$, regardless of the operating mechanism (Table 2). The efficacy of different bisphosphonates was investigated in several clinical trials to determine their value in HCM treatments (9-12). This lead to the replacement of calcitonin and glucocorticoids in the treatment of HCM by bisphosphonates which are now the preferred treatment options (8). Pamidronate was approved in 1991, and zoledronic acid was approved in 2000 for the treatment of HCM. However, results pooled from Phase III trials have shown zoledronic acid to be more potent than pamidronate with faster normalization of calcium levels, longer duration of calcium control and a higher response rate (13). In 2014, Denosumab a RANKL inhibitor has been approved for the treatment of HCM refractory to bisphosphonates with significant efficacy $(2,14)$. Approval was based on a therapy-open label one arm phase II multicenter trial of 21 patients (15). Both bisphosphonates and denosumab are also approved to reduce skeletal related events in patients with solid tumors and multiple myeloma (16). Hypercalcemia associated with parathyroid carcinoma has been more difficult to treat. Common medical approaches such as calcitonin, glucocorticoids and bisphosphonates have failed $(17,18)$. Cinacalcet, a calcimimetic was found to be effective in lowering calcium levels and maintaining them in patients with parathyroid carcinoma (19), while glucocorticoids are commonly used for the treatment of myeloma, and cancers associated with elevated calcitriol levels.

When patients and clinicians choose among the many treatments of $\mathrm{HCM}$, consideration of benefits (effectiveness evidence) and harms about patient-important outcomes are usually the main driver of the decision (20). This is currently assessed by a systematic review of benefits and harms of currently used drugs to treat the various diseases associated with hypercalcemia of malignancy. However, many other factors also affect the choice of treatment and are important for shared-decision making. The GRADE Working Group has developed an Evidence-to-Decision (EtD) framework for the assessment of factors that should complement evidence on the benefits and harms when guideline groups make recommendations. In fact, some of the treatment options involved in hypercalcemia of malignancy may be costly reaching up to $620 \$$ per dose, inconvenient and time consuming for some patients who are not able to travel to a nearby hospital for infusion (21). All of these factors should be taken into consideration when deciding on a treatment option to deliver the best care possible.

\section{Study Objectives}

The evidence to decision framework from the Grading of Recommendations, Assessment, Development and Evaluation (GRADE) Working Group (Evidence to Decision, EtD) describes five other such factors: patient's values, costs and resources, feasibility, acceptability, and equity (22). We initially used a search strategy of Epistemonikos that included key words and MESH terms capturing the key decisional factors, in the treatment of hypercalcemia of malignancy, screening the database for applicable systematic reviews (23). We were unable to find any relevant publication on the topic (Appendix 1). Therefore, and to better inform the recommendations to be made by the Endocrine Society in its Clinical Practice Guidelines on Treatment of 
Hypercalcemia of Malignancy, we decided to conduct a rigorous meta-narrative systematic review to summarize the best available evidence about these decisional factors.

\section{Methods / Design}

Due to the wide availability of different treatment options for hypercalcemia of malignancy, the aim of this systematic review is to identify important contextual and decisional factors that affect choices for therapies of hypercalcemia of malignancy in adult patients.

We have completed the search, screening and selection, and data collection stages.

\section{Information sources and search strategy}

We conducted a comprehensive search using the following online databases: Medline (OVID), Pubmed, Embase.com, the Cochrane Library and CINAHL (EBSCO). The research team developed a search strategy for each data base using MESH terms and keywords related to malignancy, hypercalcemia and factors guiding therapy decision such as patients' values and preferences, acceptability, equity, cost-effectiveness and feasibility, that was applied to adults, and limited to the last 10 years. We did not include any language restrictions. The strategy was reviewed and verified by the medical librarian at the American university of Beirut (LH), and two methodologists, at the Mayo Evidence Based Center (MHM) and the McMaster University (TP) (Appendix 2A). We also developed and executed another independent search using Medline, without any time limit, and the results were combined with the original search (Appendix $2 \mathrm{~B}$ ). We also tried to identify papers by hand searching references from the included studies..

\section{Eligibility criteria}

We included observational studies, trials, reviews and qualitative studies conducted in adult patients ( $\geq 18$ years of age) with hypercalcemia of malignancy. We included studies reporting on pharmacological therapy such as bisphosphonates, denosumab, diuretics, calcitonin, and calcimimetics as well as conservative management including hydration, avoiding calcium rich diet and vitamin D supplementation. We excluded case reports, studies conducted in the pediatric population or in patients with hypercalcemia from a condition unrelated to malignancy for example parathyroid disease, Familial Hypocalciuric Hypercalcemia $(\mathrm{FHH})$, vitamin D intoxication, and side effects of medications.

\section{Outcomes}

Our outcomes of interest are EtD factors:

- Patients or physicians values (how patients or physicians value each outcome in terms of its importance to their context and daily life)

- Cost and resources (cost effectiveness, actual charges, out of pocket costs)

- Acceptability (of treatment options and their method of administration)

- Feasibility (of the intervention as it relates to the health care environment)

- Equity (whether the intervention would exacerbate health disparities or create inequities) 
We excluded studies with inadequate outcome measurement or reporting.

\section{Study Selection}

We downloaded the literature search results into Covidence software (Covidence 2020) (24). We developed and pilot tested a screening sheet for title and abstract and another for the full texts (Appendix 3), based on our exclusion and inclusion criteria of individual studies. We performed a calibration exercise to familiarize the reviewers with the screening process.

All reviewers (AB, MR, TP, MHM, GEHF) contributed to pilot testing the screening at the title and abstract level for 100 citations. Two reviewers (AB, MR) then independently screened the remaining titles and abstracts using the screening sheet developed (Appendix $3 \mathrm{~A}$ ). We retrieved the full texts of all included citations. Two reviewers (AB, MR) screened these records independently and in duplicate using the full text screening guide (Appendix 3B). All disagreements throughout the screening process were resolved through discussion or with the help of a third reviewer as needed (TP, MHM, GEHF). All reasons for exclusion were recorded.

\section{Data collection and abstraction}

Following the full text screening, two reviewers (AB, MR) completed data abstraction independently and in duplicate using standardized data collection tables (Appendix 4). We implemented a calibration exercise to familiarize the reviewers with the process. Disagreements were resolved through discussion or with the help of a third reviewer as needed (TP, MHM, GEHF). We extracted the first author's name, date of publication and the study design, and will collect data on the characteristics, methodology and results of each of the included studies (Appendix 4). In case of any missing data, we will contact the authors of the individual studies to obtain the relevant information.

\section{Quality assessment of included studies}

The methodological quality of the included studies will be evaluated using tools appropriate for each study design, including randomized trials, cohort and case control studies, case series, and qualitative research (25-30). Quality assessment will be done independently and in duplicate.

To assess the quality of any identified RCTs, we will use the Cochrane Risk of Bias tool which assesses the following domains: bias due to sequence generation, allocation concealment, blinding of participants and personnel, blinding of outcome assessors, incomplete outcome data, and selective outcome reporting(26). To assess the quality of observational studies we will use the New Castle-Ottawa quality assessment scale assessing the following categories: Selection, comparability, and outcome(27). For case series we will assess four domains: selection, ascertainment, causality and reporting (28). Finally, for qualitative articles, we will use the CASP appraisal checklist (29).

\section{Data Synthesis}

Data will be analyzed thematically and presented narratively. Two independent reviewers will identify themes from each article until saturation and reach consensus on how the themes would converge into unique. $A$ 
third reviewer will adjudicate when consensus is not reached.

The certainty of evidence derived from the studies will be evaluated using the GRADE-CERQual approach which appraises qualitative research domains analogous to GRADE. This approach focuses on four domains: methodological limitations, coherence, adequacy and relevance (30). The overall assessment of confidence in the review findings will be based on the assessment of these individual domains.

The methodological limitations domain is assessed in individual studies based on the appropriate design, conduct, and data collection and analysis methods (31). The coherence domain assesses how clear and consistent the individual studies data are with the overall results of the review (32). The adequacy domain assesses the extent of details and available information provided in the review(33). Finally, the relevance domain assesses the extent to which the gathered individual data answers the review's objectives and questions(34).

\section{Discussion}

Treatment of hypercalcemia of malignancy is sometimes challenging due to the extensive variety of options available and wide range of benefits and harms. This systematic review will provide data on important decisional factors, which will help shape future guidelines on the management of hypercalcemia of malignancy. This study will also allow physicians and patients to decide on a therapy option based on the current evidence.

To our knowledge, this is the first systematic review conducted in hypercalcemia of malignancy to detect important decisional factors such as patient's values, costs and resources, feasibility, acceptability, and equity. The strength of this systematic review stands in its novelty, and extensive and systematic search of the literature. However, some limitations might be encountered due to the scarcity of available data and lack of reporting of our outcomes of interest.

\section{List Of Abbreviations}

HCM: Hypercalcemia of malignancy, PTHrP: Parathyroid hormone related peptide, LOH: local osteolytic hypercalcemia, PTH: Parathyroid Hormone, EtD: evidence to decision, GRADE: Grading of Recommendations, Assessment, Development and Evaluation, FHH: Familial Hypocalciuric Hypercalcemia.

\section{Declarations}

Ethical Approval and Consent to participate: Not applicable

Consent for publication: Not applicable

Availability of supporting data: Not applicable

Competing interests: The authors have no competing interests. 
Funding: None, The Endocrine Society will cover the publication fees of this protocol

Authors' contributions: All listed authors contributed substantially to the design of this protocol. All authors read and approved the final manuscript.

Acknowledgements: Dr. Aya Bassatne would like to acknowledge the training received under the Scholars in HeAlth Research Program (SHARP) that set the required foundations for a career in clinical and translational research.

\section{References}

1. Sadiq NM, Naganathan S, Badireddy M. Hypercalcemia. StatPearls. Treasure Island (FL): StatPearls Publishing Copyright (c) 2020, StatPearls Publishing LLC.; 2020.

2. Thosani S, Hu MI. Denosumab: a new agent in the management of hypercalcemia of malignancy. Future Oncol. 2015;11(21):2865-71.

3. Goldner W. Cancer-Related Hypercalcemia. J Oncol Pract. 2016;12(5):426-32.

4. Asonitis N, Angelousi A, Zafeiris C, Lambrou GI, Dontas I, Kassi E. Diagnosis, Pathophysiology and Management of Hypercalcemia in Malignancy: A Review of the Literature. Horm Metab Res. 2019;51(12):770-8.

5. Chisholm MA, Mulloy AL, Taylor AT. Acute management of cancer-related hypercalcemia. Ann Pharmacother. 1996;30(5):507-13.

6. Kandil E, Noureldine S, Khalek MA, Daroca P, Friedlander P. Ectopic secretion of parathyroid hormone in a neuroendocrine tumor: a case report and review of the literature. Int J Clin Exp Med. 2011;4(3):234-40.

7. Bhandari S, Kumar R, Tripathi P, Chan A, Mudra S, Redman R. Outcomes of hypercalcemia of malignancy in patients with solid cancer: a national inpatient analysis. Med Oncol. 2019;36(10):90.

8. Wright JD, Tergas Al, Ananth CV, Burke WM, Hou JY, Chen L, et al. Quality and Outcomes of Treatment of Hypercalcemia of Malignancy. Cancer Invest. 2015;33(8):331-9.

9. Singer FR, Ritch PS, Lad TE, Ringenberg QS, Schiller JH, Recker RR, et al. Treatment of hypercalcemia of malignancy with intravenous etidronate. A controlled, multicenter study. The Hypercalcemia Study Group. Arch Intern Med. 1991;151(3):471-6.

10. Kawada K, Minami H, Okabe K, Watanabe T, Inoue K, Sawamura M, et al. A multicenter and open label clinical trial of zoledronic acid $4 \mathrm{mg}$ in patients with hypercalcemia of malignancy. Jpn J Clin Oncol. 2005;35(1):28-33.

11. Pecherstorfer M, Steinhauer EU, Rizzoli R, Wetterwald M, Bergström B. Efficacy and safety of ibandronate in the treatment of hypercalcemia of malignancy: a randomized multicentric comparison to pamidronate. Support Care Cancer. 2003;11(8):539-47.

12. Nussbaum SR, Younger J, Vandepol CJ, Gagel RF, Zubler MA, Chapman R, et al. Single-dose intravenous therapy with pamidronate for the treatment of hypercalcemia of malignancy: comparison of 30-, 60-, and 90-mg dosages. Am J Med. 1993;95(3):297-304. 
13. Major P, Lortholary A, Hon J, Abdi E, Mills G, Menssen HD, et al. Zoledronic acid is superior to pamidronate in the treatment of hypercalcemia of malignancy: a pooled analysis of two randomized, controlled clinical trials. J Clin Oncol. 2001;19(2):558-67.

14. Dellay B, Groth M. Emergency Management of Malignancy-Associated Hypercalcemia. Adv Emerg Nurs J. 2016;38(1):15-25; quiz E1.

15. Hu MI, Glezerman IG, Leboulleux S, Insogna K, Gucalp R, Misiorowski W, et al. Denosumab for treatment of hypercalcemia of malignancy. The Journal of clinical endocrinology and metabolism. 2014;99(9):3144-52.

16. Terpos E, Ntanasis-Stathopoulos I, Dimopoulos MA. Myeloma bone disease: from biology findings to treatment approaches. Blood. 2019;133(14):1534-9.

17. de Papp AE, Kinder B, LiVolsi V, Gupta SM, Stewart AF. Parathyroid carcinoma arising from parathyroid hyperplasia: autoinfarction following intravenous treatment with pamidronate. Am J Med. 1994;97(4):399-400.

18. Mann K. Oral biphosphonate therapy in metastatic parathyroid carcinoma. Lancet. 1985;1(8420):101-2.

19. Silverberg SJ, Rubin MR, Faiman C, Peacock M, Shoback DM, Smallridge RC, et al. Cinacalcet Hydrochloride Reduces the Serum Calcium Concentration in Inoperable Parathyroid Carcinoma. The Journal of Clinical Endocrinology \& Metabolism. 2007;92(10):3803-8.

20. Gandhi GY, Murad MH, Fujiyoshi A, Mullan RJ, Flynn DN, Elamin MB, et al. Patient-Important Outcomes in Registered Diabetes Trials. JAMA. 2008;299(21):2543-9.

21. Marr HK, Stiles CR, Boyar MA, Braun TC, Hagen NA, Janzen C, et al. Feasibility of administering zoledronic acid in palliative patients being cared for in the community: results of a pilot study. Curr Oncol. 2010;17(2):69-74.

22. Alonso-Coello P, Schünemann HJ, Moberg J, Brignardello-Petersen R, Akl EA, Davoli M, et al. GRADE Evidence to Decision (EtD) frameworks: a systematic and transparent approach to making well informed healthcare choices. 1: Introduction. BMJ. 2016;353:i2016.

23. Foundation Epistemonikos. Epistemonikos [Available from: https://www.epistemonikos.org/].

24. Covidence. Covidence 2020 [Available from: www.covidence.org].

25. Viswanathan M, Patnode CD, Berkman ND, Bass EB, Chang S, Hartling L, et al. Recommendations for assessing the risk of bias in systematic reviews of health-care interventions. $\mathrm{J}$ Clin Epidemiol. 2018;97:26-34.

26. Cochrane. RoB 2: A revised Cochrane risk-of-bias tool for randomized trials 2019 [Available from: https://methods.cochrane.org/bias/resources/rob-2-revised-cochrane-risk-bias-tool-randomized-trials].

27. GA Wells BS, D O'Connell, J Peterson, V Welch, M Losos, P Tugwell,. The Newcastle-Ottawa Scale (NOS) for assessing the quality of nonrandomised studies in meta-analyses 200 [Available from: http://www.ohri.ca/programs/clinical_epidemiology/oxford.asp].

28. Murad MH, Sultan S, Haffar S, Bazerbachi F. Methodological quality and synthesis of case series and case reports. BMJ Evid Based Med. 2018;23(2):60-3. 
29. (CASP) CASP. CASP Checklist 2018 [Available from: https://casp-uk.net/wpcontent/uploads/2018/01/CASP-Qualitative-Checklist-2018.pdf].

30. CERQual G. Confidence in the Evidence from Reviews of Qualitative Research 2018 [Available from: https://www.cerqual.org/].

31. Munthe-Kaas H, Bohren MA, Glenton C, Lewin S, Noyes J, Tunçalp Ö, et al. Applying GRADE-CERQual to qualitative evidence synthesis findings-paper 3 : how to assess methodological limitations. Implementation Science. 2018;13(1):9.

32. Colvin CJ, Garside R, Wainwright M, Munthe-Kaas H, Glenton C, Bohren MA, et al. Applying GRADECERQual to qualitative evidence synthesis findings-paper 4: how to assess coherence. Implementation Science. 2018;13(1):13.

33. Glenton C, Carlsen B, Lewin S, Munthe-Kaas H, Colvin CJ, Tunçalp Ö, et al. Applying GRADE-CERQual to qualitative evidence synthesis findings-paper 5 : how to assess adequacy of data. Implementation Science. 2018;13(1):14.

34. Noyes J, Booth A, Lewin S, Carlsen B, Glenton C, Colvin CJ, et al. Applying GRADE-CERQual to qualitative evidence synthesis findings-paper 6: how to assess relevance of the data. Implementation Science. 2018;13(1):4.

\section{Tables}

ble 1: Mechanisms of hypercalcemia of malignancy and examples of their associated malignancies 
[echanism of hypercalcemia of malignancy

Jcal Osteolytic hypercalcemia
Associated malignancies

Multiple myeloma

Breast carcinoma

Leukemia

Lymphoma

Squamous cell carcinoma

Renal carcinoma

Bladder carcinoma

Breast carcinoma

Ovarian carcinoma

Prostate carcinoma

Colorectal carcinoma

Non-Hodgkin lymphoma

Leukemia

Lymphoma

Lymphomatoid

granulomatosis/angiocentric

lymphoma

Ovarian dysgerminoma

TH secreting tumors:

arathyroid Carcinoma

: Ectopic secretion of parathyroid hormone (PTH)
Ovarian carcinoma

Lung carcinoma

Neuroectodermal tumor

Neuroendocrine tumor

Thyroid papillary carcinoma

Rhabdomyosarcoma

Pancreatic carcinoma

: Hypercalcemia of malignancy treatment options 
ntional therapy

\begin{tabular}{lll}
\hline $\begin{array}{l}\text { ic saline } \\
\text { ion }\end{array}$ & Restores intravascular volume & $0.9 \% \mathrm{NaCl}$ \\
& Increases urinary calcium excretion &
\end{tabular}

\section{acological therapy}

\begin{tabular}{|c|c|c|}
\hline \multirow[t]{2}{*}{ sphonates } & Inhibit bone resorption & $\begin{array}{l}\text { IV bisphosphonates: } \\
\text { Pamidronate } \\
\text { Zoledronate }\end{array}$ \\
\hline & & $\begin{array}{l}\text { Oral bisphosphonates: } \\
\text { Clodronate } \\
\text { Ibandronate } \\
\text { Etidronate }\end{array}$ \\
\hline$\overline{u m a b}$ & Inhibits bone resorption & - \\
\hline $\min$ & $\begin{array}{l}\text { Inhibits bone resorption } \\
\text { Promotes urinary calcium excretion }\end{array}$ & - \\
\hline orticoids & $\begin{array}{l}\text { Decrease intestinal calcium absorption } \\
\text { Decrease 1,25-dihydroxyvitamin D production by } \\
\text { activated mononuclear cells }\end{array}$ & $\begin{array}{l}\text { Prednisone } \\
\text { Methylprednisone }\end{array}$ \\
\hline imetics & $\begin{array}{l}\text { Calcium-sensing receptor agonist, reduces PTH } \\
\text { synthesis and secretion }\end{array}$ & Cinacalcet \\
\hline
\end{tabular}

\section{Supplementary Files}

This is a list of supplementary files associated with this preprint. Click to download.

- Appendix1.docx

- Appendix2.docx

- Appendix3.docx

- Appendix4.docx

- PRISMAPchecklist.pdf 